\title{
Motivos de deserción estudiantil en programas virtuales de posgrado: revisión de caso y consideraciones desde el mercadeo educativo y el mercadeo relacional para los programas de retención.
}

\author{
Reasons for Student Attrition in Posgraduate Virtual Programs: Case \\ Revision and Considerations from Educational and Relational Marketing \\ for Retention Programs
}

\author{
Nubia Esther Murcia Agudelo \\ Institución Universitaria Politécnico Gran Colombiano. Colombia. \\ nmurcia@gmail.com \\ Pedro Julián Ramírez Angulo \\ Institución Universitaria Politécnico Gran Colombiano. Colombia. \\ pjramireza@unal.edu.co
}

\begin{abstract}
Resumen
El gobierno colombiano ha centrado sus esfuerzos en la educación de sus ciudadanos. Alineado con esta iniciativa, el presente trabajo pretende dar un aporte desde el mercadeo educativo y el mercadeo relacional, al fortalecimiento de la metodología virtual en el nivel post-gradual. Se utiliza un estudio cualitativo con entrevistas a profundidad hasta el nivel de saturación para conocer los motivos que inciden en la deserción y permanencia en el programa académico. Los factores de mayor repercusión encontrados fueron: los programas relacionados con los servicios académicos en los cuales se hacen relevantes la atención al estudiante, las dificultades con la plataforma y con la tecnología en general; y en segunda instancia los programas relacionados con el currículo y la instrucción en cuanto a las fallas de servicio por parte de los tutores y el currículo.
\end{abstract}

\section{Palabras clave}

Metodología virtual; retención y deserción estudiantil; mercadeo educativo y relacional; postgrado; Colombia.

\begin{abstract}
The Colombian government has focused on the education of its citizens. In line with this initiative, this paper aims at making a contribution from educational relationship marketing, to strengthen virtual methodology in the post- graduate level. A qualitative study with indepth interviews until saturation level is used to ascertain the reasons that affect attrition and retention in the academic program. The factors with greater impact found were first, those programs related to academic services which are made relevant to the attention to students, difficulties with platform and technology in general; and secondly programs related to curriculum and instruction regarding service failures by tutors and curriculum.
\end{abstract}

\section{Keywords}

Virtual methods, retention and dropout, education and relationship marketing, postgraduate, Colombia.

\section{Introducción}

Colombia ha tenido un crecimiento promedio del Producto Interno Bruto (PIB) desde el 2009 hasta el año 2012 del 5.13\% (DANE, 2012). Estas cifras se muestran alentadoras, 
de no ser por el informe de desigualdades económicas que da cuenta del desarrollo regional en la periferia del país. Las Necesidades Básicas Insatisfechas (NBI) alcanzan cifras del 79.19\% en algunos departamentos como la Amazonía, Chocó y Orinoquía desde el 2005 (Galvis, 2010). En Bogotá las NBI alcanzan el 9.2\% (DANE, 2010) y en Medellín representa el 12.42\% (DANE, 2010) lo cual requiere identificar la forma de generar crecimiento en todas las regiones del país.

Este crecimiento no se puede lograr si no se cuenta con una masa crítica de personas cualificadas y cultas que garanticen un verdadero desarrollo endógeno sostenible (Organización de las Naciones Unidas para la educación, la ciencia y la cultura, 1998). La educación superior surge como respuesta para lograr mejores condiciones de vida. Alineado con este planteamiento, el gobierno en el Plan Nacional de Desarrollo 20102014(Ministerio de Hacienda y Crédito Público, 2011) estableció la ampliación a la regionalización y flexibilidad de la oferta de educación superior, esta flexibilización da pauta para fortalecer la educación a distancia y virtual.

El Consejo Nacional de Acreditación -CNA- definió el concepto de educación a distancia como un enfoque y estrategia metodológica de organización y administración orientada a ampliar las oportunidades de acceso a la educación superior, formal y no formal, facilitando el ingreso a esta metodología educativa de un mayor número de estudiantes (Ministerio de Educación 2009). El decreto 1295 de 2010 exige como mínimo a los programas a distancia, utilizar estrategias de enseñanza -aprendizaje que superen las limitaciones espacio - tiempo entre los actores del proceso educativo; y a los programas virtuales, el uso de las redes telemáticas en un mínimo de $80 \%$ en las actividades académicas para obtener el registro calificado. (Ministerio de Educación Nacional, 2010).

A raíz de los esfuerzos, la tasa de cobertura presentó un crecimiento del $24.5 \%$ en 2002 a $37 \%$ en 2010 y el reto para el 2014 es aumentar la tasa de cobertura hasta el 50\%. El mayor número de estudiantes inscritos, matriculados y graduados se concentra en las ciudades capitales en promedio alcanza un 95\%, mientras que en los municipios no se alcanza a cubrir ni el 10\% durante la primera década del siglo (Ministerio de Educación Nacional, 2014).

El nivel de deserción: en educación presencial pasó del 30,96\% en el primer semestre de 1999 a un 13,55\% segundo semestre 2013; en el mismo período de tiempo, en educación a distancia (incluida la virtual) se presenta un nivel de deserción del $34.73 \%$ a un $20.47 \%$. (Centro Virtual de Noticias de la Educación, 2014). Como se observa la deserción afecta en mayor medida a la educación a distancia incluida la educación virtual en comparación con la educación presencial. (ACESAD, 2013; Ministerio de Educación Nacional, 2013).

Al estudiar la deserción en el nivel de posgrado de especialización, en metodología virtual, desde el 2003 al 2011 se encuentra un promedio en 56\%. Por su parte, a nivel de maestrías, según cifras del Ministerio de Educación en el año 2003 se matricularon 177 estudiantes y en 2005 la cifra de graduados fue de 33, con una deserción del $81 \%$. (Sistema Nacional de Información de la Educación Superior, 2014).

Motivos de deserción estudiantil en programas virtuales de posgrado: revisión de caso y consideraciones desde el mercadeo educativo y el mercadeo relacional para los programas de retención. . N. Murcia y J. Ramírez. 
En otros países, frente al fenómeno de la deserción, el mercadeo educativo y el mercadeo relacional, ha dado respuestas adaptativas a la medida de las necesidades de los estudiantes, aumentando el potencial de mercado, mejorando los programas educativos y reduciendo los costos de producción, entre otros puntos (Barbu, 2012). En Australia Carroll \& Birch (2013) afirman que la temática de retención de estudiantes en niveles post-graduales han recibido una atención limitada en la literatura. Bejou (2005) hace énfasis que al igual que en otros mercados de servicios, en la educación superior se requiere menos recursos para retener a los clientes existentes que reclutar a otros nuevos.

Dadas las anteriores consideraciones, el objetivo general de este trabajo de investigación es generar conocimiento entorno a los motivos de deserción estudiantil en programas virtuales de posgrado con el propósito de recomendar propuestas de intervención a nivel de mercadeo relacional y mercadeo educativo para promover la retención de estudiantes de acuerdo con la exploración de un caso específico, seleccionado bajo criterios que se especifican en la estrategia metodológica de este documento. Lo anterior involucra el cumplimiento de 3 objetivos específicos que son: 1. Indagar las proporciones de deserción en educación superior en el país en el nivel de posgrado virtual; 2. Identificar los motivos que causan deserción tanto desde los aspectos que atañen al estudiante como de los que ellos consideran son de la institución; 3. Proponer líneas de trabajo de marketing educativo y relacional en programas de retención para instituciones que cuenten con estudios de posgrado en modalidad virtual.

\section{Marco Conceptual}

\section{Mercadeo educativo}

Stachowski (2011) encontró que el marketing en educación superior es un cuerpo teórico el cual aporta a las instituciones educativas herramientas para la sobrevivencia y crecimiento en entornos competitivos. Motekaitienè, A. \& Juščius, V. (2008) afirman que el mercadeo educativo le permite a la institución educativa generar un intercambio de información a través del cual conoce necesidades y deseos de su cliente y a partir de ello debe trabajar en pro de orientar sus servicios hacia la satisfacción del cliente. Jelena Gajic (2012) busca en el mercadeo educativo entender las condiciones ambientales de las instituciones de educación superior (IES) equilibrando la oferta con demanda. Para ello propone investigar los competidores, el posicionamiento, la percepción de la calidad del servicio y la satisfacción de los usuarios. Con esta información generar estrategias al identificar nuevas oportunidades y lograr una ventaja competitiva en el mercado.

Barbu (2012) argumenta que las Instituciones de Educación Superior (IES) enfrentan varios retos: crear y mantener una buena imagen institucional, gestionar todo tipo de recursos de manera eficiente, motivar al personal académico, satisfacer las necesidades de la comunidad y obtener el apoyo de la política pública. Con tantos objetivos el mercadeo educativo ayuda a mejorar los programas educativos y a adaptar a estas organizaciones para poder responder a tal multiplicidad necesidades.

Motivos de deserción estudiantil en programas virtuales de posgrado: revisión de caso y consideraciones desde el mercadeo educativo y el mercadeo relacional para los programas de retención. . N. Murcia y J. Ramírez. 
Según Pedraza, Moreno y Pineda (2011) en Colombia aún se presenta falencias en el mercadeo educativo, dado que se observa "carencia de recursos económicos, para financiar los estudios, dificultades de tipo académico; escollos en la transición de la educación media a la universitaria, errada selección vocacional, falta de adaptación, tanto académica como social, al nuevo medio; problemas familiares y psicológicos, y el desencantamiento que producen las propuestas curriculares y las prácticas pedagógicas de los docentes". Si estos factores se presentan se genera deserción escolar.

El Ministero de Educación Nacional(2009) identifica dos conjuntos de factores que originan la deserción: el primer modelo argumenta que un estudiante toma la decisión de desertar influenciado por factores ajenos a la institución, mientras que el segundo explica que esta decisión depende del grado de integración del estudiante con el ambiente académico y social de la institución. Como se observa este fenómeno afecta tanto a estudiantes como a las instituciones educativas.

Carroll, Ng, \& Birch (2013) afirman que a nivel de postgrados en metodología virtual son pocos los estudios desarrollados, a pesar de tener mayores tasas de deserción. En el mismo estudio se dan a conocer cinco factores que influyen en la retención de estudiantes virtuales post-graduales: 1. Las respuestas brindadas por el personal académico (Lesht \& Shaik, 2005); 2. El diseño del programa (Lesht \& Shaik, 2005; Mabrito, 2012; Wang, Sierra, \& Folger, 2003), 3. El planteamiento de objetivos y metas realistas y pertinentes (Voss \& Gruber, 2006); 4. Los sistemas institucionales de apoyo (Gibson \& Graff, 1992; Simpson, 2004); 5. La orientación vocacional dada para escoger el programa (Chandler, Levin, \& Levin, 2002; Derby \& Smith, 2004; Rowley, 2003).

Finalmente, se mencionan tres factores que influyen en la retención del estudiante: 1. la disposición (actitud) fortalece o disminuye la motivación del estudiante (Lauer, 2002), 2. Planteamiento de objetivos e intenciones realistas (Seidman, 2005; Voss \& Gruber, 2006; Watson, Johnson, \& Austin, 2004); 3. La satisfacción del estudiante frente a su programa (DeShields, Kara, \& Kaynak, 2005; Douglas, Douglas, \& Barnes, 2006; Longden, 2002; Seidman, 2005; Taylor, 2005).

\section{Mercadeo relacional}

El concepto de mercadeo relacional fue creado en la década de 1980 y trabajado desde diferentes autores. Estos autores crearon diferentes definiciones del mismo pueden apoyar y complementar las perspectivas expuestas desde el mercadeo relacional. Por ejemplo Greenberg (2008) afirma que el mercadeo relacional es una fusión de procesos en el cual se incluye el negocio y las políticas de nivel global empresarial, cuya finalidad es captar, retener, y dar servicio a los clientes. Greenberg(2008) al respecto concluye que es un conjunto coherente y completo de procesos y tecnologías las cuales buscan gestionar las relaciones con clientes actuales y potenciales y con asociados de la empresa. Estas relaciones de desarrollan, a través de los departamentos de mercadeo, ventas y servicio, con independencia del canal de comunicación... la meta de CRM (Customer Relationship Management) es optimizar la satisfacción de los clientes y asociados, las ventas de la empresa y su eficiencia, construyendo las relaciones más fuertes posibles en el nivel organizativo.

Motivos de deserción estudiantil en programas virtuales de posgrado: revisión de caso y consideraciones desde el mercadeo educativo y el mercadeo relacional para los programas de retención. . N. Murcia y J. Ramírez. 
Kotler, P., Kartajaya, H. and Setiawan, I. (2010) afirman que el mercadeo ha pasado de estar centrado en el producto (marketing 1.0) a centrarse en el consumidor (Marketing 2.0) y luego a girar en torno a la esencia humana (marketing 3.0) donde lo primordial es la defensa de los valores, como lo son el servicio público, la sostenibilidad, entre otros. Para Schnarch (2012) el mercadeo relacional busca la fidelización del cliente, a través del establecimiento de relaciones de acuerdo al perfil, la necesidad y el hábito de cada cliente, llevándolo a relaciones uno a uno, es decir, a generar una relación personalizada.

Alcaide (2010) manifiesta que la relación con el cliente debe convertirse en el corazón de la gestión empresarial fortaleciendo y consolidando relaciones a largo plazo buscando la fidelización, trabajando desde la perspectiva del cliente. Según este autor, la estrategia relacional implica una visión global e integral que debe caracterizar todas las relaciones, contactos, interacciones, comunicaciones, que establece una empresa con base en sus clientes. Llevando el mercadeo relacional al sector educativo, según Suka (2011) se debe identificar y cualificar a los clientes actuales y potenciales, conociendo sus necesidades, con esta información se pueden adaptar los programas de mercadeo y los servicios educativos controlando y gestionando la relación con cada cliente.

\section{Deserción y retención de estudiantes}

La deserción, según Giovagnoli (2002) se puede entender como una situación a la que se enfrenta un estudiante cuando aspira y no logra concluir su proyecto educativo en el tiempo planeado. UNESCO - Ieselac (2010) en su Glosario de la educación superior en Latinoamérica y el Caribe define la deserción como "la proporción de estudiantes que suspenden, cambian de carrera, o la abandonan antes de obtener el título." (http://www.iesalc.unesco.org.ve), citado por (Torres, 2012.).

Para la Universidad Nacional de Colombia (2005) la deserción es una interrupción en la trayectoria académica individual, la cual genera un distanciamiento entre las expectativas del estudiante frente a su proyecto educativo y las posibilidades objetivas de llevarlo a cabo. El MEN (2009) identifica como desertor, desde el punto de vista de las IES (Institución de Educación Superior) cualquier estudiante que abandona la institución. A su vez, clasifica la deserción en: precoz cuan es admitido y no se matricula; temprana, cuando se retira en los primeros semestres y tardía cuando abandona en los últimos semestres la carrera escogida.

Como respuesta a la deserción se ha fomentado la retención escolar, "el término retención se deriva del latín retentio-onis, que significa acción y efecto de retener" (Pedraza, Moreno, \& Pineda, 2011). La OEA define retención como: "la capacidad que tiene el sistema educativo para lograr la permanencia de los alumnos en las aulas, garantizando la terminación de ciclos y niveles en los tiempos previstos y asegurando el dominio de las competencias y conocimientos correspondientes" Agencia Interamericana para la Cooperación y el Desarrollo (AICD) (2003). El MEN (2010) define la retención como la "trayectoria académica exitosa que lleva a un estudiante que se matricula a la educación superior a su graduación".

Motivos de deserción estudiantil en programas virtuales de posgrado: revisión de caso y consideraciones desde el mercadeo educativo y el mercadeo relacional para los programas de retención. . N. Murcia y J. Ramírez. 
Desde el mercadeo educativo se han presentado distintas propuestas para promover la retención escolar, como las siguientes: Pinto (2007) añade la importancia del ajuste que experimenta el estudiante de la vida de colegio a la vida universitaria incluida las exigencias administrativas e institucionales, además de las personales.

\section{Mercadeo educativo relacional}

Suka (2011) afirma que el mercadeo educativo relacional es el conjunto de planes y programas estratégicos para el logro de metas y objetivos en un tiempo determinado estableciendo relaciones estrechas y de fidelidad con los estudiantes. Alineado con esta perspectiva se encuentra el modelo creado por Swail, Redd y Perna (2003) modelo desde la cual se desarrollará este estudio. Es importante aclarar que este modelo es citado por Pedraza, Moreno, \& Pineda (2011)

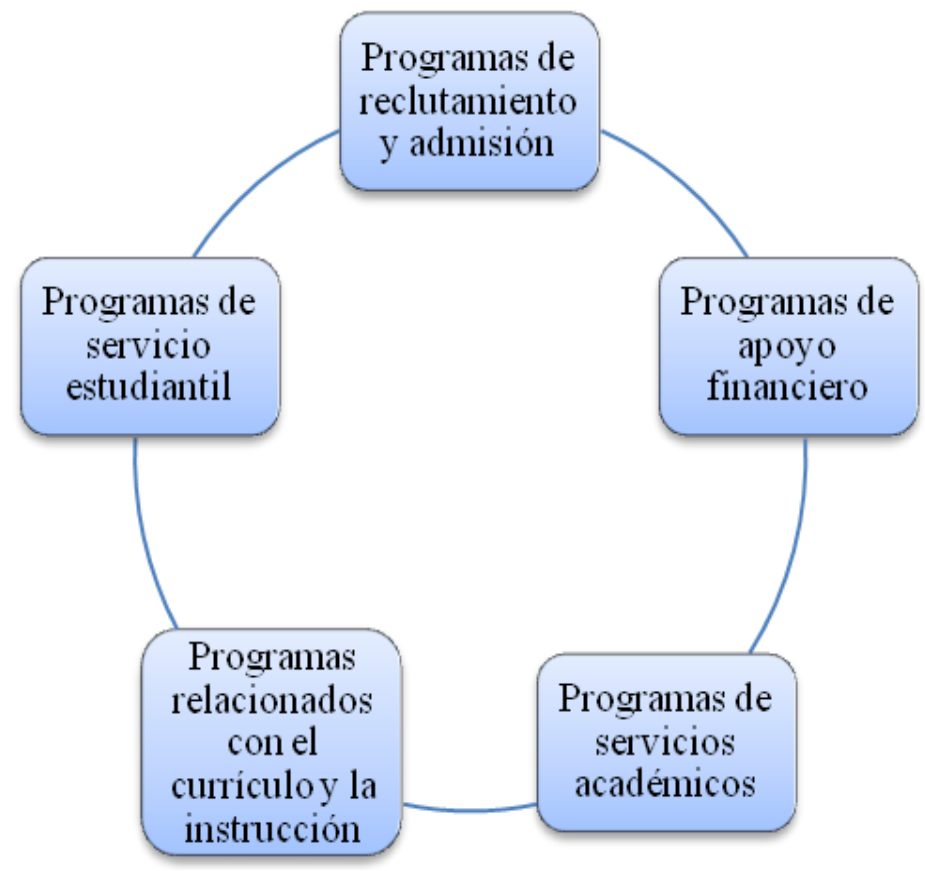

Tomado de: (Pedraza, Moreno, \& Pineda, 2011).

Programas de reclutamiento y admisión: compuesto por las acciones informativas y de orientación que intentan, en primera instancia, ubicar a aquellos estudiantes cuyos valores y metas mantienen cierto grado de coherencia con los de la institución, y que, en segundo lugar, pretenden nivelar esos valores y metas para que la transición y el ajuste al medio universitario revistan un carácter menos drástico y traumático. (Pedraza, Moreno, \& Pineda, 2011).

Programas de apoyo financiero: busca facilitar la financiación de los estudios y a subvencionar gastos asociados a la supervivencia del estudiante (Pedraza, Moreno, \& Pineda, 2011). A 2011 el costo promedio de la educación de un hijo en Colombia equivale al $65 \%$ del PIB por habitante. El $28 \%$ corresponde al valor de la matrícula y el resto a costos de sostenimiento. En Suecia, las familias destinan el $4 \%$ del PIB por habitante al pago de la matrícula, en México el 15\%, en Francia el 6\%, en USA el 26\%, razón por la cual es tan importante el apoyo económico. (Isaza, 2011)

Motivos de deserción estudiantil en programas virtuales de posgrado: revisión de caso y consideraciones desde el mercadeo educativo y el mercadeo relacional para los programas de retención. . N. Murcia y J.

Ramírez. 
Programas de servicios académicos: orientados a enfrentar de manera prioritaria, aunque no siempre exclusiva, las falencias académicas de los estudiantes. En él se encuentran los apoyos académicos como tutorías, monitorias, respuestas solicitadas por el estudiante y respondidas oportunamente y toda clase de acompañamientos para el logro de los objetivos académicos. (Pedraza, Moreno, \& Pineda, 2011).

Programas relacionados con el currículo y la instrucción: examinar la calidad de los currículos y las prácticas pedagógicas, la calidad del cuerpo docente. (Pedraza, Moreno, \& Pineda, 2011).

Programas de servicio estudiantil: incluyen programas cuya intención es promover la vinculación de los estudiantes con su medio, de tal manera que se den los ajustes para una efectiva integración social. En él se encuentran todos los programas que invitan al estudiante a participar en actividades extra-curriculares y le permiten generar relaciones con estudiantes de la misma institución. (Pedraza, Moreno, \& Pineda, 2011).

Rivera-Camino \& Molero Ayala (2010) incluyen seis componentes: orientación de los estudiantes, la orientación de los trabajadores, de orientación competidor, orientación de la empresa donante, orientación medio ambiente, y la coordinación interfuncional; Voon (2008) agrega es importante centrar los esfuerzos en identificar las creencias, comportamientos y procesos interfuniconales para comprender de forma global las necesidades del cliente y birndar un excelente servicio;(De Shields, Kara, \& Kaynak, 2005) incluyen la satisfacción de los estudiantes con su experiencia del curso es determinante para optar por retirarse o no de sus estudios, (Carroll, Birch, \& Ng, 2013) agregan que el soporte dado desde el área de sistemas a los estudiantes hacen parte de los factores que influyen en la retención de estudiantes.

\section{Método}

Se desarrolló un estudio exploratorio con abordaje cualitativo (Hernández, Fernández, \& Baptista, 2010) de orden deductivo con respecto a la elaboración del instrumento, con un muestreo por saturación (Alonso, 2003) utilizando la técnica de entrevista semiestructurada a profundidad para recopilar la información de los motivos de deserción estudiantil. Cabe destacar que el instrumento fue validado por dos expertos en mercadeo y por dos directivos de una institución de educación superior, uno de ellos dedicado al área virtual y el otro a programas de retención como asesor del Ministerio de Educación Nacional.

El instrumento fue aplicado para el Programa de la Especialización en Gerencia de Proyectos en Inteligencia de Negocios en la Institución Universitaria Politécnico Gran Colombiano, el cual fue escogido luego de estudiar las estadísticas de programas de especialización en la institución e identificar cuál de ellos tenía un mayor nivel deserción. Es importante dar cuenta de que el método de caso en la investigación puede ser utilizado para aplicar teoría en situaciones dadas, retroalimentarla, generar nuevas hipótesis y realizar procesos inductivos (Álvarez, Enriquez, \& Iturbe, 2005).

Se exploraron dos grupos de estudiantes, el primer grupo sigue sus módulos con regularidad y el segundo son personas que decidieron abandonar el programa. En ambos

Motivos de deserción estudiantil en programas virtuales de posgrado: revisión de caso y consideraciones desde el mercadeo educativo y el mercadeo relacional para los programas de retención. . N. Murcia y J. Ramírez. 
grupos se tomaron como categorías orientadoras las desarrolladas en el modelo de Swail, Redd y Perna, desde el cual se desarrolló este estudio, y se buscaron cuáles fueron los motivos tanto para continuar como para abandonar a nivel de programa, de metodología virtual e institución universitaria en general.

Adicionalmente, las entrevistas continuaron hasta que no se presentaron aportes sustanciales con el fin de cumplir con el muestreo por saturación. Asimismo todas las fases de la ejecución metodológica de este trabajo cumplen con los criterios de rigor en el ámbito de lo cualitativo: dependencia, credibilidad auditabilidad, transferibilidad (Salgado, 2007).

Para el análisis de la información se utilizó Atlas TI y se realizó codificación abierta con respeto a las preguntas formuladas, codificación axial con respecto a los ejes de indagación del instrumento y familias asociadas a las categorías orientadoras generadas en la literatura.

\section{Ficha técnica}

\begin{tabular}{|l|l|}
\hline $\begin{array}{l}\text { Población } \\
\text { objetivo }\end{array}$ & $\begin{array}{l}\text { Hombres y mujeres mayores de 18 años que hayan cursado } \\
\text { módulos de especializaciones bajo la metodología virtual, en } \\
\text { los últimos 3 años. }\end{array}$ \\
\hline Técnica & $\begin{array}{l}\text { Entrevistas semiestructurada a profundidad, por medio } \\
\text { telefónico. }\end{array}$ \\
\hline Participantes & $\begin{array}{l}\text { El criterio de saturación se cumplió cuando se entrevistaron } \\
\text { alumnos que están o estuvieron vinculados al programa de la } \\
\text { Especialización en Gerencia de Proyectos en Inteligencia de } \\
\text { Negocios en la Institución Universitaria Politécnico Gran } \\
\text { Colombiano, 9 en total, 5 activos (personas que has seguido } \\
\text { sus estudios desde el primer módulo sin interrupciones) y } \\
\text { estudiantes que han desertado (individuos que en un punto de } \\
\text { sus estudios de especialización suspendieron su continuidad } \\
\text { académica). }\end{array}$ \\
\hline $\begin{array}{l}\text { Momento } \\
\text { estadístico }\end{array}$ & \begin{tabular}{l} 
18 de octubre - 20 de octubre de 2014. \\
\hline Financiación
\end{tabular} \\
\hline $\begin{array}{l}\text { Persona } \\
\text { natural que la } \\
\text { realizó }\end{array}$ & Nubia Esther Murcia Agudelo. \\
\hline
\end{tabular}

Nota: Tabla de elaboración propia.

\section{Resultados}

En este análisis se utilizó la transcripción de las entrevistas y su posterior estudio con la herramienta estadística Atlas Ti. A continuación se da cuenta de las respuestas de los estudiantes frente a los programas de reclutamiento y admisión, apoyo financiero, currículo e instrucción, servicio estudiantil, servicio académico y otras variables que no son parte del modelo de Swail, Redd y Perna, pero que fueron nominadas por los participantes.

Motivos de deserción estudiantil en programas virtuales de posgrado: revisión de caso y consideraciones desde el mercadeo educativo y el mercadeo relacional para los programas de retención. . N. Murcia y J.

Ramírez. 


\section{Programas de reclutamiento y admisión}

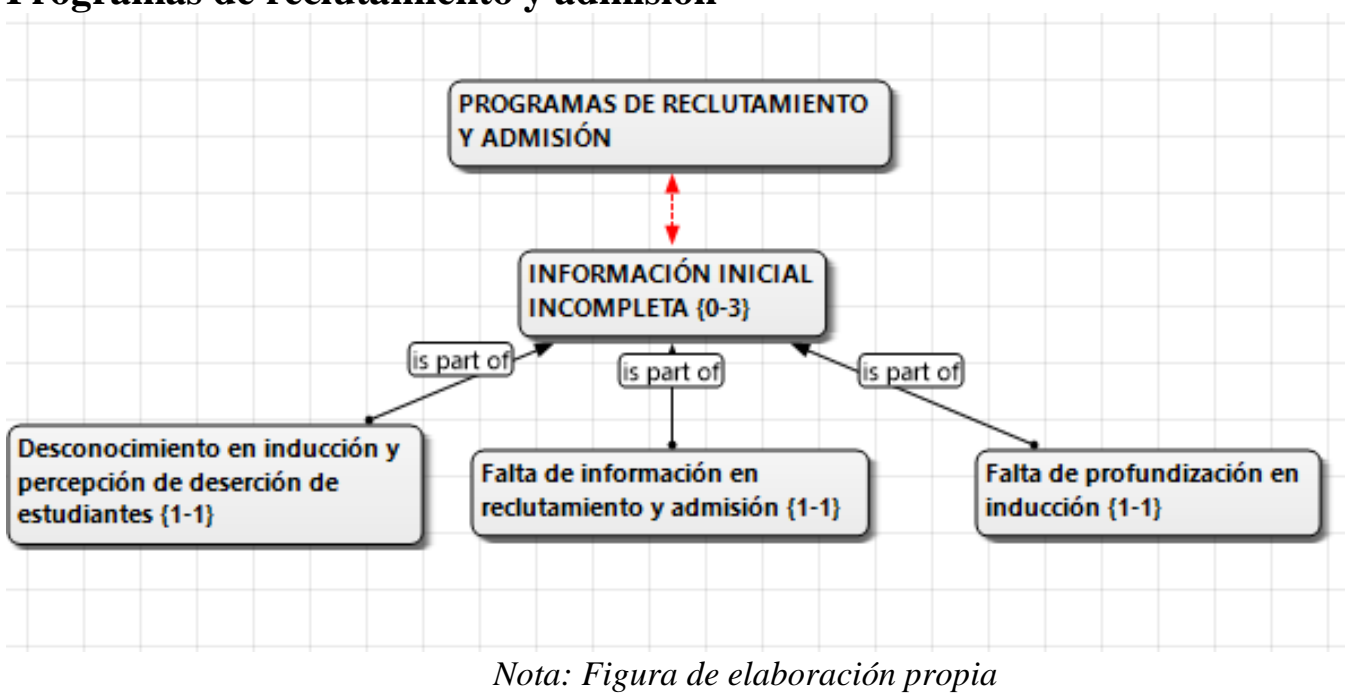

Frente a los programas de reclutamiento y admisión los estudiantes manifiestan falta de información completa recibida por el estudiante. Los participantes afirman: "yo hablé con la persona Politécnico antes escribirme, ellos dicen cuando uno se contacta: eso es una nota, es lo último en tecnología, no te preocupes por nada te vas a poder comunicar... eso es solo para cogerte la plata"... "Yo le decía: mira en mi caso es la primera vez que voy a estudiar virtual solamente hecho unos cursos cortos. Ella me decía: no te preocupes es súper sencillo"; "decía esto es última maravilla del mundo, van estudiar para aprender, un carretas y me quedé esperando a que lo que me dijeron funcionara así...", entre otros comentarios.

\section{Programas de apoyo financiero}

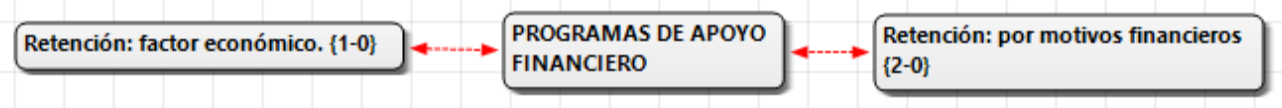

Nota: Figura de elaboración propia

Los programas de apoyo financiero recibieron comentarios que favorecen la retención dado que los estudiantes afirman no querer perder la inversión e identifican que el costo de la especialización está acorde a lo recibido: "el retiro me afectó a nivel económico más que todo porque yo ya había adelantado un pago, A nivel personal es desmotivante porque uno quiere empezar algo para terminarlo", entre otros.

\section{Programas relacionados con el currículo y la instrucción}

Motivos de deserción estudiantil en programas virtuales de posgrado: revisión de caso y consideraciones desde el mercadeo educativo y el mercadeo relacional para los programas de retención. . N. Murcia y J.

Ramírez. 


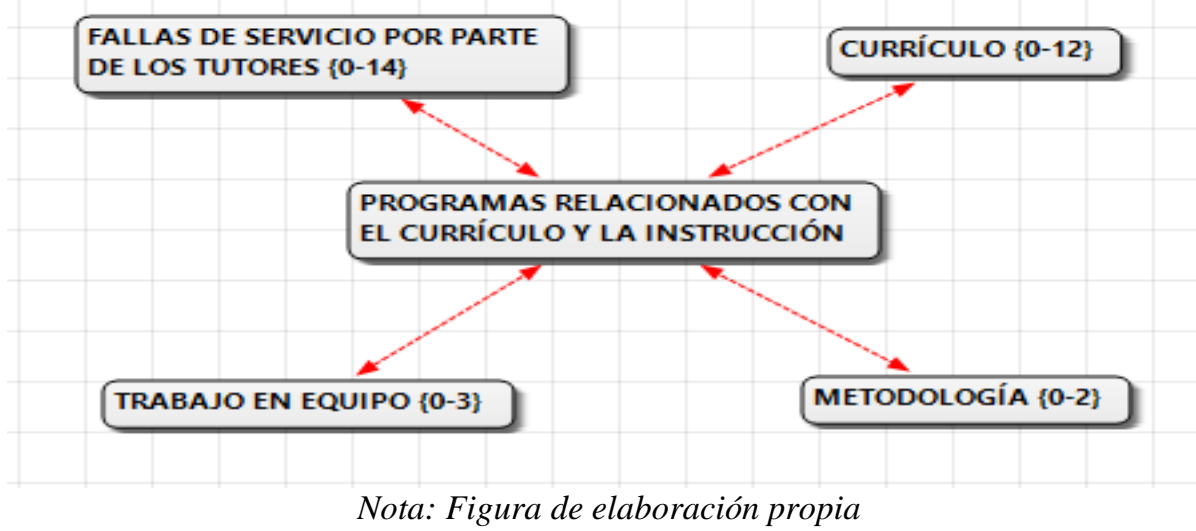

Se observa que los programas relacionados con el currículo y la instrucción, son los más comentados, asociadas con dificultades en la tutoría y dificultades académicas con los contenidos de las materias. En segunda instancia se mencionan las dificultades con la metodología y con la elaboración de trabajos en grupo.

Escuchando los comentarios de los estudiantes su mayor queja se presenta cuando el tutor no les responde los correos, no les califica a tiempo, no reciben la retroalimentación suficiente y la institución responde de manera tardía a las quejas, inquietudes, solicitudes y necesidades de los estudiantes.

Frente a lo anterior se evidencian verbalizaciones como: "la especialización tienen mucho contenido de sistemas, bueno, para mí fue sencillo, era entendible porque yo soy ingeniero de sistemas, pero para alguien de mercadeo o de publicidad... las bases de datos o minería de datos son materias complicadas"; "la idea es ver lo de negocios"; "el grupo dice lo mismo, te dicen que quieren ver ya lo de proyectos porque tiene mucho tiempo viendo otras cosas y quieren ver la parte en concreto de proyectos... porque hemos visto muchas materias de bases de datos y algo de estrategias liderazgo, financiera que si obviamente se necesita, pero vimos tres materias que es de base de datos y en este momento lo que quiero es empezar ya a ver metodologías de proyectos"; entre otros.

Pareciera que el estudiante no tiene claridad de la malla curricular o posee una expectativa distinta a la vivenciada en el orden de presentación de los módulos.

Algunos estudiantes consideran que los docentes no tienen la suficiente experiencia para desempeñarse como tutor a nivel de especialización. En menor cantidad, algunos comentan ser afectados por la no respuesta de mensajes enviados a sus profesores y en general, se sienten solos en el proceso educativo, aunado a la falta de un acompañamiento más intensivo en aquellas materias que no son de manejo común entre todos los estudiantes, como lo es, por ejemplo, la destreza en programación y manejo de bases de datos.

Entre las verbalizaciones al respecto se encuentran: "tuvimos problemas con unos maestros, por ejemplo con la materia de sistemas de información el profesor solo sacó una nota, y cada semana el asistía a la clase pero realmente se dedicaba solamente a mostrar unas diapositivas y eso realmente lo puedo hacer por Internet"; "los profesores nos dicen: envíenos un correo, nosotros les contestamos, pero pasa con muchos que uno les escribe y nunca responden, entonces uno queda en el limbo y toca realizar la entrega

Motivos de deserción estudiantil en programas virtuales de posgrado: revisión de caso y consideraciones desde el mercadeo educativo y el mercadeo relacional para los programas de retención. . N. Murcia y J. Ramírez. 
correspondiente... el día de la nota se ven las consecuencias de realizar esas entregas de carreras... uno corre toda la semana y es tremendo"; "en una materia no me calificaron un trabajo porque tuve problemas con el correo, escribí muchas veces, nunca me lo calificaron a pesar de mis comunicaciones y haber aclarado la situación, a varios compañeros les pasó lo mismo".

Hay quienes no están de acuerdo con la evaluación grupal como forma estandarizada de evaluación, desde su punto de vista, limita al estudiante en su desempeño y manejo autónomo del tiempo: "como son trabajos compartidos nos coordinamos por Skype, por teléfono, por correo, uno hace un esfuerzo inmenso para sacar la plata y comunicarse con los compañeros... es un tema bien complejo"; "solicitan muchos trabajos en equipo, por lo cual se requiere coordinar con varias personas, que tienen ocupaciones al igual que uno y no es fácil ponernos de acuerdo, entonces la elaboración de documentos y trabajos en equipo es complejo y se pierde mucho tiempo, me parece que en ese tipo de estudios, el trabajo en grupo no tiene cabida".

Otros por el contrario, afirman que el trabajo en equipo les ha beneficiado porque el compartir con personas de distintas profesiones permite el aporte interdisciplinario y favorece la elaboración de los documentos finales solicitados como medio de evaluación en los módulos: "Realmente me ha ido bien en los trabajos en grupos, estoy con tres ingenieros de sistemas, entonces en la parte de programación ellos son fuertes y eso ha facilitado que aprenda lo de bases datos".

\section{Programas de servicios académicos}

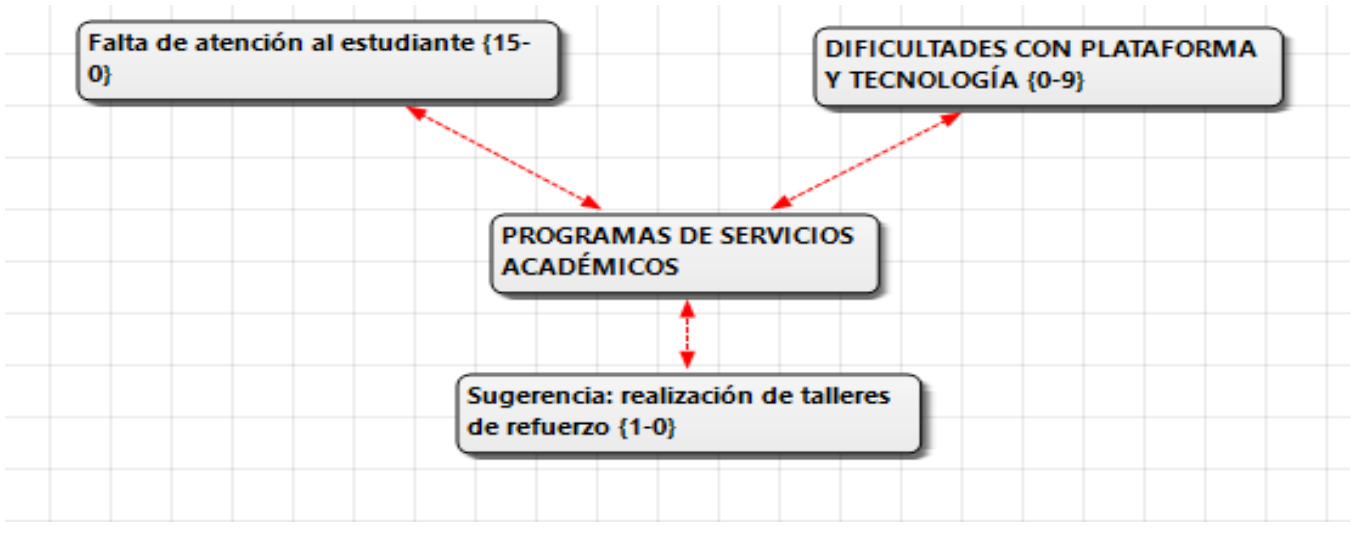

Nota: Figura de elaboración propia

Los participantes manifiestan falta de atención al estudiante, dificultades en plataforma, etc. Entre las verbalizaciones se encuentran: "a muy pocos les enviaron el recibo del segundo pago con tiempo, a algunos les llegaron vencidos, a otros no les llegó, a mí nunca me llego, yo me enteré porque todos hablaban del pago, entonces quienes faltábamos empezamos a llamar para avisar que no nos habían enviado recibo, eso creó incertidumbre, no sabíamos si era que nos iban a sacar, es un desorden tremendo y no hay canales de comunicación reales, eso fomenta que el grupo esté tan reducido, la gente se aburre y no vuelve "; "el Politécnico debe tener una persona que sea capaz de direccionar al alumno de forma clara, deberían tener instructivos donde se manifiesten como se manejan las cosas y crear canales de comunicación confiables y fáciles"; "de la

Motivos de deserción estudiantil en programas virtuales de posgrado: revisión de caso y consideraciones desde el mercadeo educativo y el mercadeo relacional para los programas de retención. . N. Murcia y J.

Ramírez. 
universidad me llamaron hace como dos meses a ver si pagaba el otro semestre, y no se habían dado cuenta que yo había dejado de asistir, no hay un buen seguimiento", estas y otras afirmaciones son planteadas por los participantes.

Es relevante observar que los servicios académicos se encuentran en segundo orden de recordación por parte del estudiante. Se observa también en el estudio, información no vinculada directamente dentro de las variables mencionadas por el modelo de Swail, Redd y Perna, las cuales fueron incluidas en: otras variables que afectan la retención y otras variables que afectan la deserción.

\section{Causas de deserción / retención ajenas a la institución}

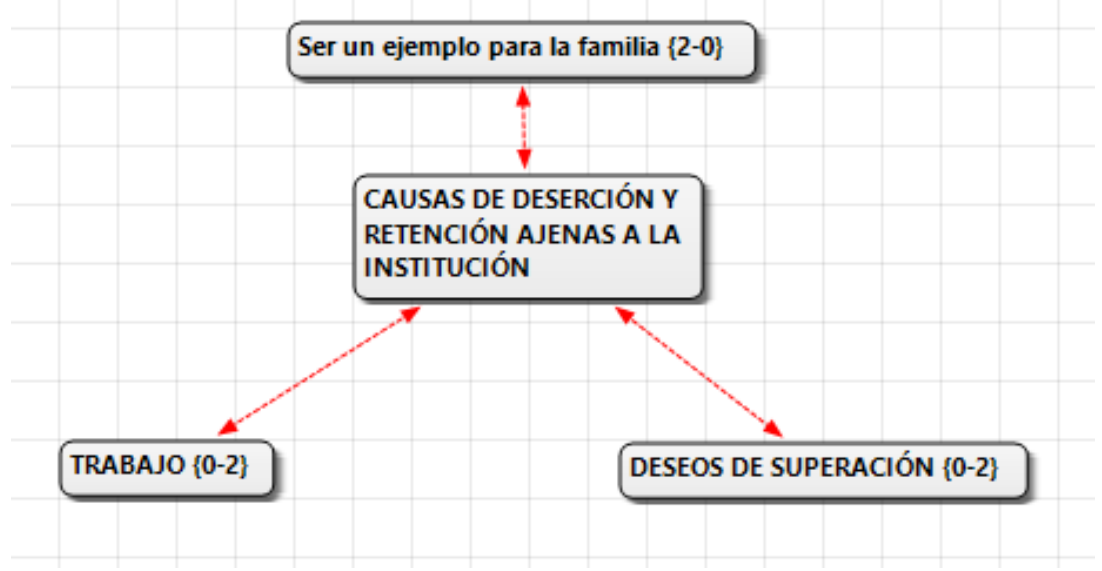

Nota: Figura de elaboración propia

En pro de la retención se observa el deseo de superación reflejado en terminar y graduarse, las expectativas por las siguientes materias y la familia apoyan la decisión de seguir estudiando la especialización. Dentro de las características con mayor acogida está el respeto por el manejo de tiempo individual en la modalidad virtual, el cual según afirman pueden manejar su tiempo, dedicar el tiempo de traslado a estudiar e incluso optimizar el tiempo de compartir en la familia: "me gusta el tiempo que tengo, O sea que evito desplazamientos hacia otro lugares cuando salgo del trabajo, voy directamente a mi casa y puedo estudiar tranquila"; "yo puedo manejar el tiempo como yo quiera, tengo tiempo de hacer los trabajos y todo lo que me piden en el momento en que yo quiera, sé cuáles son las fechas de entrega y era lo que yo quería, no tener que asistir al lugar físicamente, poder estudiar y manejar mi tiempo", entre otros.

Por otra parte se identificaron variables que promueven la deserción. Es contradictorio con los argumentos anteriores, lo afirmado por otros estudiantes, desde su perspectiva existen limitaciones de tiempo: "al trabajar con otras personas el tiempo ya no es tanto de uno, debe coordinar con otros... uno busca este tipo de formación para poder trabajar con el tiempo de uno". Se encuentran también tensiones entre las exigencias académicas versus los compromisos laborales: "a veces me dicen que tengo comité y como es un club, la gente que compone las juntas y los Comités directivos se reúnen generalmente después de las 5:00, 6:00 7:00 pm la noche y me toca salir a las 10 u 11 de la noche, y a esa hora sale uno cansado", entre otros.

Motivos de deserción estudiantil en programas virtuales de posgrado: revisión de caso y consideraciones desde el mercadeo educativo y el mercadeo relacional para los programas de retención. . N. Murcia y J.

Ramírez. 
Finamente es importante anotar frente al modelo que los programas de servicios académicos no tuvieron verbalizaciones.

En resumen, los programas con mayor fuerza en recordación e impacto para los estudiantes están relacionados con la familia de los servicios académicos y en segunda instancia con la familia de programas relacionados con el currículo y la instrucción, las otras familias no dan cuenta de una influencia fuerte en los resultados.

\section{Conclusiones}

Este trabajo permitió generar conocimiento entorno a los motivos de deserción estudiantil en programas virtuales de posgrado, de tal manera se observa como en Colombia ha crecido en un $12,5 \%$ el ingreso de estudiantes a educación medida en tasa de cobertura se ha concentrado en las ciudades principales, aunque los niveles de deserción aún están en el orden de $34.7 \%$ en educación presencial y de $20.47 \%$ en educación virtual (Centro Virtual de Noticias de la Educación, 2014). Desafortunadamente en estudios virtuales post-graduales la deserción se encuentra en un promedio del 56\%. (Sistema Nacional de Información de la Educación Superior, 2014).

Los motivos de deserción más representativos arrojados por el estudio involucran los programas relacionados en primera instancia con los servicios académicos en los cuales se hacen relevantes la atención al estudiante y las dificultades con la plataforma y la tecnología y en segunda instancia los programas relacionados con el currículo y la instrucción en cuanto a las fallas de servicio por parte de los tutores y el currículo. Lo cual coincide con los aportes de Swail, Redd y Perna (2003).

A la luz de los anterior, el estudiante día a día espera un contacto más personalizado de las instituciones prestadoras de servicios educativos, en donde pudiese entablar una comunicación bidireccional, con la cual anhela obtener respuestas a sus inquietudes, dudas, preguntas y/o problemáticas, a la medida de cada situación, encontrándose en el mercadeo educativo y relacional una herramienta fundamental para afianzar la retención de estudiantes, lo cual está alineado con planteamientos como los de Schnarch (2012); Suka (2011) y Alcaide (2011).

Se observa que el apoyo en los programas relacionados con el currículo y la instrucción deben enfocarse principalmente en generar un seguimiento más estricto al cumplimiento por parte de los tutores que apoyan el proceso de aprendizaje de los estudiantes, así como el generar respuestas más diligentes frente a las dificultades que se presentan con la plataforma en general con la tecnología. Resultados que reafirman las argumentaciones planteadas por Swail, Redd y Perna (2003).

A nivel curricular las estrategias de intervención pueden encaminarse en dos frentes. En primera instancia aclarar desde los primeros contactos con el estudiante cuales son las exigencias temáticas, el pensum y los pre-requisitos académicos con los cuales debe contar el estudiante para abordar las exigencias académicas de la especialización, y en segunda fortalecer los espacios de retroalimentación, no obligatorios, con los docentes, con el fin de apoyar mejor al estudiante en materias que no son de su ámbito profesional, pero que hacen parte del programa académico al cual se vinculó.

Motivos de deserción estudiantil en programas virtuales de posgrado: revisión de caso y consideraciones desde el mercadeo educativo y el mercadeo relacional para los programas de retención. . N. Murcia y J. Ramírez. 
Resultados que dan mayor sustento a las argumentaciones planteadas por Swail, Redd y Perna (2003).

Aunque no son numerosas las verbalizaciones acerca de los programas de reclutamiento y admisión, el estudiante al inscribirse dentro de una especialización busca terminar sus estudios y graduarse, para ello requiere desde el inicio conocer cuáles son las exigencias, el programa y las exigencias a nivel de conocimientos previos. Ello coincide con los postulados como los de Seidman (2005) y al modelo de Swail, Redd y Perna (2003).

Si bien las categorías orientadoras se basan en al modelo de Swail, Redd y Perna este trabajo revela la importancia de la familia denominada: otras causas de deserción / retención ajenas a la institución como el deseo de superación, la familia y el trabajo, los cuales pueden llevar al estudiante a desistir o continuar trabajando para graduarse, dentro de ellos se encuentra fortalecida la posición de Lauer (2002)

Es interesante observar como los servicios estudiantiles no tuvieron verbalizaciones, lo cual se identifica como relevante a la hora de tomar decisiones frente a las acciones a realizar. Este resultado es el único que va en contravía a lo planteado por Swail, Redd y Perna (2003).

Analizando la influencia entre las carencias mostradas de los servicios académicos y su afectación con la variable económica, algunos estudiantes identifican deficiencias de comunicación entre el área académica y docente puesto que los recibos de pago no son recepcionados por los estudiantes de forma oportuna e incluso algunos que se han retirado o han suspendido su actividad académica son contactados para el cobro del siguiente semestre. Al mismo respecto, algunos estudiantes que se han retirado temporalmente manifiestan el no tener respuesta a la solicitud de conocer como vincularse nuevamente como estudiante. Características encontradas en los estudios de Swail, Redd y Perna (2003); Isaza (2011); Pedraza, Moreno, \& Pineda (2011), entre otros.

Se identificó que la variable financiera aparece al momento de decidir mantenerse como estudiantes activos puesto que el no perder el dinero invertido se concibe solo si se obtiene el título. Ello lleva al estudiante a generar esfuerzos adicionales que son parte del mantenerse estudiante como lo es el manejo del tiempo; sin embargo estos esfuerzos de acuerdo a su percepción aunque valiosos se suman a esfuerzos no tangibles como el manejo del tiempo, entre otros, corroborando lo encontrado por Pedraza, Moreno y Pineda (2011).

La retención de estudiantes, puede generar en términos financieros mayores beneficios económicos para la institución dado que el estudiante busca una vinculación a largo plazo, en la cual se culmine el objetivo deseado, por ello, en la medida en que el estudiante permanezca, el ingreso permanece de acuerdo a lo presupuestado, para ello se requiere inversión principalmente en tres frentes: académico, tecnológico y de servicio personalizado. Planteamiento que coincide con las afirmaciones de Greenberg (2008).

Las estrategias de retención que genera cada institución educativa impactan a nivel de desarrollo social y económico del país, en virtud de ello, el fomento de la educación en

Motivos de deserción estudiantil en programas virtuales de posgrado: revisión de caso y consideraciones desde el mercadeo educativo y el mercadeo relacional para los programas de retención. . N. Murcia y J. Ramírez. 
la modalidad virtual, es vital para el crecimiento nacional, puesto que pueden brindar educación y apoyar el desarrollo en aquellos lugares donde, por diferentes circunstancias la educación presencial no cubre esas necesidades.

Lo anterior confirma lo presentado por postulados presentados, entre otros por Galvis (2010); UNESCO (1998) y el Ministerio de Educación Nacional (2014).

\section{Propuestas para mejorar la retención estudiantil}

Frente a los hallazgos encontrados en el transcurso de las entrevistas, se podría recomendar a las instituciones que administran posgrados virtuales el diseño de equipos por programa, donde se monitoreen las alertas académicas las siguientes pautas que buscan influenciar la percepción del estudiante de forma positiva, siguiendo el modelo de Swail, Redd y Perna, (2003):

\section{Mejoras relacionados con el currículo y la instrucción}

Para complementar la labor de seguimiento realizada, puede asignarse un auxiliar por cada grupos que desarrolle un programa, el cual esté a cargo del monitoreo de plataforma indicando a las instancias competentes las novedades que se están presentando en cada materia (ausencia de respuestas a los estudiantes por parte de docentes, calificaciones tardías, no ingreso a la plataforma luego de 48 horas de cualquier docente, quejas planteadas en los espacios interactivos comunes como foros de participación, entre otras).

Promocionar las capacitaciones extra-curriculares, sesiones de chat y foros que se ofrecen a los estudiantes, incentivando incluso con notas extra por la participación (no solo asistencia), a los estudiantes que generen preguntas y aprendizaje. Los estudiantes pueden enviar incluso previamente, preguntas al espacio de chat par que a partir del material solicitado se inicie la sesión. Este material puede quedar disponible en la web para quien desee acceder a él y fortalecer su conocimiento.

Es importante aclarar que la asistencia sería de carácter voluntario. Con respecto a los trabajos en equipo, podría asignarse mayor peso evaluaciones de carácter individual y menor peso en la calificación al trabajo en equipo, además de identificar en las cuales materias podrían no desarrollar sus desempeños finales de forma grupal.

Estas propuestas se desarrollan desde la perspectiva de Swail, Redd y Perna, (2003) donde se identifica que la calidad del currículo y los apoyos económicos impactan en la permanencia estudiantil, en conjunto con los enfoques de: Lesh \& Shaik (2005); De Shields, Kara\& Kaynak, (2005); Douglas, Douglas, \& Barnes (2006); Longden (2002); Seidman (2005); Taylor (2005); Lesht \& Shaik, (2005); Lesht \& Shaik (2005); Mabrito (2012); Wang, Sierra, \& Folger (2003); Pedraza, Moreno y Pineda (2011) y Rivera Camino \& Molero Ayala (2010).

\section{Servicio académicos}

Motivos de deserción estudiantil en programas virtuales de posgrado: revisión de caso y consideraciones desde el mercadeo educativo y el mercadeo relacional para los programas de retención. . N. Murcia y J. Ramírez. 
Aprovechando la presencia de los auxiliares mencionados en el programa anterior, estas personas tendrían a cargo además: estar atentas a la suspensión de actividades de los estudiantes a lo largo de los módulos y buscar contactarlos para identificar si por parte de la universidad se puede apoyar al estudiante o si desea pedir aplazamiento y retomar sus estudios más adelante; atender las dudas, inquietudes o solicitudes que el estudiante realice a nivel administrativo o tecnológico, como son: problemas de plataforma, trámites de pago, generación de certificaciones, aplazamientos entre otras.

Toda esta información el auxiliar remite a la persona encargada para que así las directivas a cargo puedan generar respuestas oportunas y efectivas dentro de los 3 días hábiles siguientes a los imprevistos que se presenten, si ello no ocurre, autorizar a este auxiliar a escalar el caso al siguiente nivel jerárquico, optimizando la búsqueda de respuesta al estudiante.

Las propuestas presentadas frente al servicio estudiantil devienen de: Swail, Redd y Perna, Greenberg (2008); Alcaide (2011); Carroll, D.; Birch, D. \& Ng, E. (2013); Gibson \& Graff(1992); Rivera-Camino \& Molero Ayala (2010) y Simpson, (2004).

\section{Reclutamiento y admisión}

Ampliar la capacitación de la fuerza de ventas en: 1. El conocimiento del contenido temático y 2. La comunicación de los programas a vender. Los puntos anteriores incluyen conocer las contenidos de las materias y los conocimientos básicos que requiere el estudiante para cursar la especialización.

Por otra parte, se propone identificar si el candidato realmente está entendiendo y atento a la información ofrecida a través de la tonalidad y el contenido de las respuestas que emite o si es importante reforzar o ampliar en algún punto, además de poder enviar al futuro estudiante a su correo el link del sitio web donde encuentra a malla curricular y breves pero completas presentaciones de los módulos a cursar.

Para afianzar que la información se presente de forma completa, la comisión por venta debe estar atada, no solo a la matrícula del estudiante sino también a la validación de la entrega de información completa al candidato.

Frente a reclutamiento y admisión se observa que Swail, Redd y Perna, (2003); Seidman (2005); Voss \& Gruber, (2006); Watson, Johnson, \& Austin, (2004); Chandler, Levin, \& Levin, (2002); Derby \& Smith, (2004); Rivera - Camino \& Molero Ayala (2010); Rowley, (2003) generan sustento al respecto y dan soporte a los planteamientos aquí expuestos.

\section{Apoyo financiero}

Generar espacios de comunicación y verificación de estudiantes activos entre las áreas académica, financiera y de mercadeo y ventas en donde se crucen las bases de datos de los estudiantes, corroborando su estado actual (vigente, retirado o suspendido) de forma anticipada al final de los cortes, con lo cual se pueda generar el envío de recibos con antelación y disponer de personas direccionadas a reincorporar aquellas personas interesadas en volver a estudiar.

Motivos de deserción estudiantil en programas virtuales de posgrado: revisión de caso y consideraciones desde el mercadeo educativo y el mercadeo relacional para los programas de retención. . N. Murcia y J. Ramírez. 
En este punto se identificaría con ayuda de los auxiliares propuestos en las sugerencias de los programas de servicio estudiantil, elaborar bases de datos de quienes se han retirado, quienes continúan y quienes desean volver a vincularse, de este último grupo conocer el motivo de aplazamiento o retiro de la universidad como insumo para direccionar de forma más efectiva al estudiante para volver a vincularse a su actividad académica.

Siendo una variable identificada como positiva para la retención de estudiantes, son importantes los aportes de: Swail, Redd y Perna (2003); Isaza (2011) y Pedraza, Moreno y Pineda (2011) para la generación de aportes en la materia.

\section{Limitaciones y futuras líneas de investigación}

Por medio de esta investigación se conoció el impacto de las variables estudiadas en estudiantes universitarios de postgrado con metodología virtual, través de un estudio cualitativo. Partiendo de este punto, sería interesante desarrollar investigaciones cuantitativas que podrían validar si los hallazgos de esta investigación son generalizables a otros contextos post-graduales.

Por lo anterior, se sugiere incentivar en otros programas e instituciones, este tipo de investigaciones ampliando el conocimiento de la temática, en pro del cumplimiento de los objetivos individuales, institucionales y en general de crecimiento a nivel país y realizar un estudio a largo plazo para conocer el impacto del crecimiento intelectual y profesional de los estudiantes en las regiones apartadas, vs. el crecimiento de la economía. Estos estudios podrían iniciarse en departamentos como Chocó, Amazonía, etc. en los cuales Galvis encontró mayores índices de NBI.

Una línea de investigación a futuro estaría enmarcada en la indagación específica de los apoyos financieros y como ello ha influido en la consecución de los objetivos finales de los estudiantes.

Otra investigación que desde lo encontrado se propone, es el corroborar con estudios estadísticos cuantitativos si existe relevancia o no, para el estudiante vinculado con programas virtuales de post-grado, programas relacionados con servicios estudiantiles.

Sería interesante implementar y conocer si las propuestas presentadas tuvieron efecto en la retención de los estudiantes y si fue así, el grado de deserción de los estudiantes, corroborando si fue reducido o no al implementar dichas prácticas.

En línea con lo anterior, si el impacto de las iniciativas fue positivo, replicar en otros niveles de educación para observar si los resultados pueden ser generalizables a estudiantes de pregrado.

Generar un mayor análisis del porque no se observa recordación cuando de temas de servicio académico se trata, conocer si el estudiante de postgrado a metodología virtual le gustaría contar con mayor énfasis en las propuestas que desde este punto puedan devenir o no.

Motivos de deserción estudiantil en programas virtuales de posgrado: revisión de caso y consideraciones desde el mercadeo educativo y el mercadeo relacional para los programas de retención. . N. Murcia y J. Ramírez. 
Abordando una perspectiva distinta se propone indagar la postura de los dueños de las instituciones educativas frente a los trabajos realizados frente a la temática estudiada y su interés por ponerlos a prueba. Esta indagación podría ser interesante, generando además las proyecciones financieras a largo plazo y su correspondiente comparación con inversiones en otros mercados.

Otra arista interesante para profundizar en la temática es la posición de los rectores, directores de áreas administrativas y académicas y personal de apoyo institucional, con respecto a la permanencia de los estudiantes. Las preguntas a resolver serían: ¿percibe que su papel en la dinámica institucional es importante para fortalecer la permanencia del estudiante hasta finalizar su ciclo propedéutico?; ¿piensa que su área recibe el apoyo tecnológico, administrativo y económico necesario para hacer una buena labor?, entre otras inquietudes que de estos estudios se generen.

Fin de redacción del artículo: diciembre de 2014

Murcia, N. y Ramírez, P. (2015). Factores asociados a la deserción estudiantil en programas virtuales: consideraciones desde el mercadeo educativo y el mercadeo relacional. RED, Revista de Educación a Distancia. 45(6). Consultado el (dd/mm/aaaa) en http://www.um.es/ead/red/45

\section{Bibliografía}

Alcaide, J. C. (2010). Fidelización de clientes. Madrid: ESIC.

Alonso, L. E. (2003). La mirada cualitativa en sociología: una aproximación interpretativa. Madrid: Fundamentos.

Álvarez, I., Enriquez, J., \& Iturbe, E. (2005). Los estudios de caso como estrategia para la formación en Gestión de Experiencias del Sector Educativo. México: Taller Abierto.

Barbu, A. (2012). Analysis of mercadeo tools and activities within educational services organizations, in order to increase their efficiency. Annals of the University of Oradea, Economic Science Series, 1106-1111.

Bejou, D. (Marzo - Abril. de 2.005). Treating students like customers. Recuperado el 10 de Agosto. de 2.014, de AACSB Internacional: http://www.aacsb.edu/publications/Archives/marapr05toc.asp\#sthash.BKdgQyXT.dpuf

Carroll, D., Ng, E., \& Birch, D. (2013). Strategies to Improve Retention of Postgraduat Business Students in Distance Education Courses: An Australian Case. Turkish Online Journal of Distance Education, 140-153.

Carroll, D., Ng, E., \& Birch, D. (Enero de 2013). Strategies to improve retention of postgraduate business studentes in distance education courses: an Australian Case. Turkish Online Journal of Distance Education-TOJDE., 14(1), 140-155.

Motivos de deserción estudiantil en programas virtuales de posgrado: revisión de caso y consideraciones desde el mercadeo educativo y el mercadeo relacional para los programas de retención. . N. Murcia y J. Ramírez. 
Centro Virtual de Noticias de la Educación. (2014). IES colombianas recibirán apoyo de Mineducación para enfrentar la deserción estudiantil.Bogotá: CVNE.

Chandler, M., Levin, J., \& Levin,. S. (2002). The evolution of an online community of distributed learners/teachers. Paper presented at the 2002 American Educational. New Orleans.

DANE. (13 de 9 de 2010). DANE. Recuperado el 19 de 7 de 2014, de Boletín Censo General

PerfilBogotá:http://www.dane.gov.co/files/censo2005/perfil_pdf_cg2005/11001t7t00 $0 . p d f$

DANE. (13 de 9 de 2010). Departamento Administrativo Nacional de Estadística. Recuperado el 19 de 7 de 2014, de Boletín General. Censo 2005. PerfilMedellín.:http://www.dane.gov.co/files/censo2005/perfil_pdf_cg2005/05001t7t 000.pdf

DANE. (2012). Comunicado de prensa. Producto interno bruto: cuarto trimestre y total anual 2011.Bogotá: DANE.

Derby, D., \& Smith, T. (2004). An orientation course and community college retention. Community College Journal of Research and Practice, , 763-773.

DeShields, O. W., Kara, A., \& Kaynak, E. (2005). Determinants of business student satisfaction and retention in higher education: Applying Herzberg's two-factor theory. International Journal of Educational Management, 9 (2), 129-139.

DeShields, O., Kara, A., \& Kaynak, ,. E. (2005). Determinants of business student satisfaction and retention in higher education: Applying Herzberg's two-factor theory. International Journal of Educational Management., 14 (3), 251-267.

Douglas, J., Douglas, A., \& Barnes, ,. B. (2006). Measuring student satisfaction at a UK university. Quality Assurance in Education,, 14 (3), 251-267.

Gajic, J. (2012). Importance of mercadeo mix in higher education institutions. Singidunum Journal Of Applied Sciences, 29-41.

Galvis, L. A. (Enero de 2010). Persistencia de las desigualdades regionales en Colombia : Un análisis espacial La serie Documentos de Trabajo Sobre Economía Regional es una publicación del Persistencia de las desigualdades regionales en Colombia: un análisis espacial. Recuperado el Junio de 2013, de Banco de la República: http://www.banrep.gov.co/docum/Lectura_finanzas/pdf/DTSER-120.pdf

Galvis, L. A. (2010). Persistencia de las desigualdades regionales en Colombia: Un análisis espacial La serie Documentos de Trabajo Sobre Economía Regional es una publicación del Persistencia de las desigualdades regionales en Colombia: un análisis espacial. Documentos de trabajo sobre economía regional, 1-44.

Motivos de deserción estudiantil en programas virtuales de posgrado: revisión de caso y consideraciones desde el mercadeo educativo y el mercadeo relacional para los programas de retención. . N. Murcia y J.

Ramírez. 
Giovagnoli, P. (2002). Determinantes de la deserción y graduación universitaria: una aplicación utilizando modelos de duración. Documento de trabajo 37. La Plata: Universidad Nacional de la Plata.

Greenberg, P. (2008). CRM: Gestión de Relación con los Clientes. Madrid: McGrawHill.

Hernández,, R., Fernández, , C., \& Baptista, M.P. (2010). Metodología de la investigación. Mexico: Mc Graw Hill.

Isaza, J. (31 de Agosto de 2011). Cobertura en la educación superior. El Espectador.

Kotler, P., Kartajaya, H. \&Setiawan, I. (2010).Marketing 3.0: From Products toCustomers to the Human Spirit, New Jersey: John Wiley \& Sons, Inc.

Lauer, C. (2002). Enrolments in higher education: Do economic incentives matter? Education + Training., 44(4/5), 179-185.

Lesht, F., \& Shaik, N. (2.005). Best practices in helping students complete online degree. Paper presented at the 19th Annual Conference on Distance Teaching and Learning. Madison, WI.

Lesht, F., \& Shaik, N. (2005). Best practices in helping students complete online degree. Paper presented at the 19th Annual Conference on Distance Teaching and. Madison, WI.

Lesht, F., \& Shaik, N. (s.f.). Best practices in helping students complete online degree programs. Paper presented at the 19th Annual Conference on Distance Teaching and Learning. Madison, WI.

Longden, B. (2002). Retention rates: Renewed interest but ¿whose interest is being served? Research Papers in Education, 17 (1), 3-29.

Mabrito, M. (22 de October 22, de 2012.). Guidelines for establishing interactivity in online courses. Obtenido de http://www.innovateonline.info/pdf/vol1_issue2/Guidelines_for_Establishing_Intera ctive

Ministerio de Educación Nacional. (21 de 03 de 2014). Sistema para la Prevención de la Deserción en las Instituciones de Educación Superior. Recuperado el 21 de 03 de 2014, de SPADIES: http://www.graduadoscolombia.edu.co:8380/eportal/web/snies/capital-o-municipiodel-domicilio-de-la-institucion

Ministerio de Educación Nacional. (2009). Deserción Estudiantil en la Educaicón superior Colombiana. Metodología de seguimiento, diagnóstico y elementos para su prevención. Bogotá: Imprenta Nacional de Colombia.

Motivos de deserción estudiantil en programas virtuales de posgrado: revisión de caso y consideraciones desde el mercadeo educativo y el mercadeo relacional para los programas de retención. . N. Murcia y J.

Ramírez. 
Ministerio de Educación Nacional. (18 de 03 de 2013). Sistema para la Prevención de la Deserción en las Instituciones de Educación Superior. Recuperado el 04 de 04 de 2014, de Spadies: http://menweb.mineducacion.gov.co/seguimiento/estadísticas

Ministerio de Educación Nacional. (2014). Sistema Nacional de Información de Educación Superior. Recuperado el 12 de Octubre de 2014, de SNIES: http://snies.mineducacion.gov.co/consultasnies/programa/buscar.jsp?control=0.1635 208104908893

Ministerio de Educación Nacional. (04 de 04 de 2014). Sistema para la Prevención de la Deserción en las Instituciones de Educación Superior . Recuperado el 04 de 04 de 2014, de Spadies: http://spadies.mineducacion.gov.co/spadies/JSON.html

Ministerio de Educación Nacional. (20 de Abril de 2010). Decreto 1295 por el cual se reglamenta el registro calificado de que trata la Ley 1188 de 2008. Bogotá, Colombia.: Imprenta Nacional de Colombia.

Ministerio de Educación Nacional. (2014). Sistema Nacional de Información de la Educación Superior. Recuperado el 13 de Octubre de 2014, de SNIES: http://www.mineducacion.gov.co/sistemasdeinformacion/1735/w3-article-

212400.html

Ministerio de Educación Nacional. (29 de abril de 2014). Sistema para la Prevención de la Deserción es las instituciones de Educación Superior. Recuperado el 29 de abril de 2014, deSPADIES: http://www.graduadoscolombia.edu.co:8380/eportal/web/snies/capital-o-municipiodel-domicilio-de-la-institucion

Ministerio de Educación. (18 de Marzo de 2013). Estadísticas de Educación Superior. Recuperado el 30 de Junio de 2014, de www.mineducacion.gov.co/.../1735/articles212350_resumen.xls

Ministerio de Hacienda y Crédito Público. (16 de junio de 2011). Ley 1450 del 16 de junio de 2011 por la cual se expide el plan nacional de desarrollo, 2010-2014. Ley 1450 del 16 de junio de 2011 por la cual se expide el plan nacional de desarrollo, 2010-2014. Bogotá, Colombia.: Imprenta Nacional de Colombia.

Ministerio de Educación Nacional. (18 de Marzo de 2013). Sistema para la Prevención de la Deserción en las Instituciones de Educación Superior. Recuperado el 2014 de Marzo de 24, de Spadies: http://spadies.mineducacion.gov.co/spadies/JSON.html

Ministero de Educación Nacional. (2009). Deserción Estudiantil en la Educación Superior Colombiana. Metodología de seguimiento, diagnóstico y elemntos para su prevención.Bogotá.: Imprenta Nacional de Colombia.

Motekaitienè, A. and Juščius, V. (2008) "Specifics of Marketing in the Higher Education System", Social Research, Vol. 12, No. 2, pp. 97-103.

Motivos de deserción estudiantil en programas virtuales de posgrado: revisión de caso y consideraciones desde el mercadeo educativo y el mercadeo relacional para los programas de retención. . N. Murcia y J.

Ramírez. 
Organización de las Naciones Unidas para la educación, la ciencia y la cultura. (1998). La educación superior en el siglo XXI, visión y acción. Paris: UNESCO.

Organización de los Estados Americanos, Agencia Interamericana para la Cooperación y el Desarrollo (AICD). (2003). Documento base del proyecto estrategias y materiales pedagógicos para la retención escolar. Argentina: Ministerio de Educación, ciencia y Tecnología.

Pedraza, A., Moreno, I., \& Pineda, C. (2011). Efectividad de las estrategias de retención universitaria: la función del docente1. Educaicón y Educadores, 14(1), 119-135.

Pineda-Báez, C. P.-O. (2011). Efectividad de las estrategias de retención universitaria: la función del docente. Educación Y Educadores, 119-135.

Pinto, M. D. (2007). Cuestión de supervivencia. Graduación, deserción y rezago en la Universidad Nacional de Colombia.Bogotá: Unibiblos.

Rowley, J. (2003). Retention: Rhetoric or realistic agendas for the future of higher education. The International Journal of Educational Management., 17(6), 248-253.

Salgado, A. C. (2007). Investigación cualitativa: diseños, evaluación del rigor metodológico y retos. Liberabit. Revista de Psicología, 13, 71-78.

Schnarch, A. (2012). Researchin emotion and affect in the history of education, History of Education. Journal of the History of Education Society, 689-695.

Seidman, A. (2005). Minority student retention: Resources for practitioners. New Directions for Institutional Research, , 125, 7-24.

Simpson, O. (2004). The impact on retention of interventions to support distance learning students. Open Learning., 19 (1), 79-95.

Sistema Nacional de Información de la Educación Superior. (21 de 03 de 2014). Recuperado el 21 de 03 de 2014, de Spadies: http://menweb.mineducacion.gov.co/seguimiento/estadisticas/principal.php?seccion= $12 \&$ id_categoria $=1 \&$ consulta $=$ mat_meto\&nivel $=12 \&$ dpto $=\& e t=\&$ mun $=\& i n s=\&$ sede $=$

Stachowski, C. A. (2011) "Educational Marketing: A Review and Implications forSupporting Practice in Tertiary Education", Educational Management Administration \& Leadership, Vol. 39, No. 2, pp. 186-204.

Suka, R. (2011). Relación del Mercadeo Estratégico Relacional Educativo en la calidad de la Gestión Pedagógica en las Instituciones Educativas de la Red 07 en la Unidad Gestión Educativa Local 05 en San Juan de Lurigancho - Lima 2009. Relación del Marketing Estratégico Relacional Educativo en la calidad de la Gestión Pedagógica en las Instituciones Educativas de la Red 07 en la Unidad Gestión Educativa Local

Motivos de deserción estudiantil en programas virtuales de posgrado: revisión de caso y consideraciones desde el mercadeo educativo y el mercadeo relacional para los programas de retención. . N. Murcia y J.

Ramírez. 
05 en San Juan de Lurigancho - Lima 2009. Lima., San Juan de Lurigancho, Perú: Universidad Nacional Mayor de San Marcos.

Taylor, R. (2005). Creating a connection: Tackling student attrition through curriculum. Journal of Further and Higher Education., 29(4), 367-374.

Torres, L. (2012). Retención estudiantil en la educación superior. Revisión de la literatura y elementos de un modelo para el contexto colombiano. Bogotá: Pontificia Universidad Javeriana.

UNESCO. (1998). La educación superior en el siglo XXI, visión y acción. Paris: UNESCO.

Universidad Nacional de Colombia. (2005). Cartilla de Bienestar Universitaro.Bogotá., Colombia.: Dirección de Bienestar Universitario.

Voss, R., \& Gruber, T. (2006). The desired teaching qualities of lecturers in higher education: A means end analysis. Quality Assurance in Education, 14(3), 217-242.

Wang, M., Sierra, C., \& Folger, T. (2003). Building a dynamic online learning community among adult learners. Educational Media International, 40(12), 49-61.

Watson, G., Johnson, G., \& Austin, M. (2004). Exploring relatedness to field of study as an indicator of student retention. Higher Education Research and Development, 23(1), 57-72. 